\title{
Multisolitons with vector mesons on the two-sphere
}

\author{
F. L. Carrasco ${ }^{*}$ and Oscar A. Reula \\ FaMAF-Universidad Nacional de Córdoba, IFEG, CONICET, Ciudad Universitaria, \\ 5000 Córdoba, Argentina
}

(Received 2 October 2013; published 5 August 2014)

\begin{abstract}
Recent studies have suggested a strong connection between the static solutions of the 3D Skyrme model and those corresponding to its low-dimensional analog (baby-Skyrme model) on a two-sphere. We have found almost identical solutions considering an alternative two-dimensional model in which a vector meson field is introduced and coupled to the system, instead of the usual Skyrme term. It has been known that including this vector meson field in three dimensions stabilizes the nonlinear sigma model without the need for a term that is quartic on derivatives of the pion fields (Skyrme term). The resulting model has proven to share many of the features that the usual Skyrme theory has, but with a better mathematical formulation in terms of the well posedness of its evolution equations. In the present work, we have numerically searched for static multisolitonic solutions of this alternative stabilization, for the case in which the base space is a two-sphere. Moreover, we analyze the stability of these solutions under small perturbations in a fully dynamical setting. We have also considered the inclusion of a particular potential term in the Lagrangian and explored the low- and high-density phases of solitons for different ranges of the parameter space, achieving solitons localized enough, which allows for a comparison with planar (two-dimensional) studies.

DOI: 10.1103/PhysRevD.90.045007

PACS numbers: 12.39.Dc, 04.25.D-
\end{abstract}

\section{INTRODUCTION}

A sigma model is a field theory, where the field takes values on a Riemannian manifold, that is, a map from a spacetime into a target space, usually a symmetric space. The nonlinear sigma model is one of the simplest systems admitting static topological soliton solutions, which can be characterized by the degree of the map: an integer $B$ that in the "field theory language" is known as the topological charge of the field configuration. Identifying this scalar field (or map) with a pion field in three spatial dimensions, the different topological solitonic solutions can be interpreted as baryons.

However, the topologically nontrivial static solutions are dynamically unstable, and the model requires the inclusion of extra terms in the Lagrangian in order to yield stable solutions. With only pion degrees of freedom, modification is attained by adding to the free Lagrangian the so-called Skyrme term, which is quartic in derivatives. This leads to the well-known Skyrme model [1].

Within the Skyrme model, a soliton with topological charge 1 is called a Skyrmion, which, when suitably quantized constitutes a model for a physical nucleon, while solitons of higher topological charges (multi-Skyrmions) are classical models for heavier nuclei. This idea of representing nucleons as solitons of the effective pion field remains attractive even in the context of QCD, and moreover, the approach becomes exact in the large $N_{c}$ limit [2].

\footnotetext{
*fcarrasc@famaf.unc.edu.ar

†reula@famaf.unc.edu.ar
}

It was later realized that it is possible to stabilize the nonlinear sigma model without this fourth order term by coupling the baryon current to a $\omega$ meson field [3]. This new model has a few important advantages over the original one. The Skyrme model, being a quasilinear system of equations, has propagation speeds which depend on the solution, and they can, for some initial data, become imaginary, thus destroying the well posedness of its evolution and the predictive power of the theory. On the other hand, the vector meson model is a semilinear, symmetric hyperbolic system of equations. Thus, all of its propagation speeds are proportional to the speed of light. Later extensions of this new proposal to stabilize the sigma model have considered multisolitons in three spatial dimensions [4] and in its two-dimensional analog [5]. Besides computing the stationary solutions, studies also exist on the dynamical aspects of the model, such as Refs. [6,7]. In these studies it is observed that the solutions of the vector meson theory have very similar properties to the ones of the Skyrme model. That is, in spite of being mathematically very different theories, they seem to share many common features in their solution spaces.

Topological solitons are usually studied in flat space, but there are various reasons why the curved-space setting is interesting, the most important one being the emergence of a new length scale on the problem, namely, the one of the underlying geometry. This allows for an interesting interplay between this new scale and the size of the solitons. Physically, this allows one to model a finite density of solitons, as well as the transition between the high-density and the low-density phases. It has been realized that many of the qualitative results of lattice calculations could be 
obtained in a much easier way by studying the behavior of a few solitons on a compact manifold $[8,9]$. This feature has several potential applications in condensed matter physics (e.g. Refs. $[10,11])$.

In this work, we study the alternative stabilization of the sigma model by means of the inclusion of a $\omega$ vector meson, in its two-dimensional version on a unit sphere. We will numerically look for the static multisoliton solutions of this model and compare them with the ones found in [12] for the baby-Skyrme model, where a strong connection between the symmetries of their solutions (on the sphere) and those of the 3D Skyrme model was pointed out. We are also interested in a comparison with those solutions obtained in [4] for the 3D vector meson theory. Additionally, we want to further explore the role of the ratio (size of the solitons)/(radius of the sphere) on the possible static configurations of the model.

We begin by describing the model in some detail in Sec. II. Section III discusses the initial configurations considered, as well as details of our numerical implementation. In Sec. IV we show the results of our computational studies. First, we treat the case in which no potential term is present, like the one in Ref. [12] for the baby-Skyrme model. Then, we include a potential term into the Lagrangian and analyze the interplay of the two length scales from the resulting theory. We summarize and conclude with some further comments in Sec. V.

\section{FORMALISM}

\section{A. The model}

Our starting point is the nonlinear sigma model from $S^{2}$ to $S^{2}$, that is, a map $\phi$ between a spacetime $\left(\mathbb{R} \times S^{2}, g\right)$ and a target Riemannian space $\left(S^{2}, H\right)$, where $g_{a b}=h_{a b}-n_{a} n_{b}$ ( $n_{a}$ being the normal to the $t=$ const homogeneity hypersurfaces) and with both $h_{a b}$ and $H_{A B}$ representing the metric of the unit sphere. We will use capital letters to denote indices on the target space and lowercase letters to represent spacetime indices.

The action is given by the integral (over a spacelike hypersurface) of the trace of the pullback of the Riemannian metric (target space) into the spacetime (base space):

$$
S(\phi):=\frac{1}{2} \int_{\Sigma_{t}} g^{a b} \nabla_{a} \phi^{A} \nabla_{b} \phi^{B} H_{A B} d V .
$$

The topological degree of the map is an integer $B$ that characterizes the field configuration at a given time. It is defined as the pullback of the surface element of the target $S^{2}$, integrated over the physical space and normalized by the total area,

$$
B=\operatorname{deg}[\phi]:=\frac{1}{4 \pi} \int_{\Sigma_{t}} \Omega_{a b}
$$

where $\Omega_{a b}:=\frac{1}{2} \nabla_{a} \phi^{A} \nabla_{b} \phi^{B} \varepsilon_{A B}$ is, as mentioned, the pullback of the surface element $\epsilon_{A B}$ of the target manifold.

Since the degree of the map $B$ is an integer-valued quantity, it turns out that it must be conserved throughout the evolution, assuming the dynamics to be smooth (i.e., no singularities develop). This also follows directly from the conservation of the topological current $B^{a}$ (i.e., $\nabla_{a} B^{a}=0$ ), which is just the Hodge dual of the two-form $\Omega_{a b}$,

$$
B^{a}:=-\frac{1}{4 \pi} \epsilon^{a b c} \Omega_{b c} .
$$

It might be convenient to think of the map $\phi$ as taking values on $\mathbb{R}^{3}$ but constrained to the sphere. That is, we consider a map to $\mathbb{R}^{3}, \phi^{A}$, where the index $A$ takes values $A=1,2,3$ and with $\phi^{A} \phi_{A}:=\phi_{1}^{2}+\phi_{2}^{2}+\phi_{3}^{2}=1$.

In this constrained formulation, the Lagrangian density reads

$$
\mathcal{L}_{\sigma}=\frac{1}{2} g^{a b}\left(\nabla_{a} \phi^{A}\right)\left(\nabla_{b} \phi^{B}\right) \delta_{A B}+\frac{1}{2} \lambda\left(1-\phi^{2}\right)
$$

where $\lambda$ is the Lagrange multiplier enforcing the constraint.

Following Refs. [3,5], and in order to stabilize the solitonic solutions of this theory, we introduce a vector meson field $\omega$ coupled through the baryon current and obtain the Lagrangian density of the model:

$$
\begin{aligned}
\mathcal{L}:= & \mathcal{L}_{\sigma}+V(\vec{\phi})+\frac{1}{2} \nabla_{a} \omega_{b}\left(\nabla^{a} \omega^{b}-\nabla^{b} \omega^{a}\right)+\frac{1}{2} M^{2} \omega_{a} \omega^{a} \\
& +g \omega_{a} B^{a}
\end{aligned}
$$

where $M$ is the mass of the meson field and $g$ is the coupling constant. We have also included a potential term $V$ which only depends on the pion field, $\phi^{A}$.

\section{B. Equations of motion}

Variations of the action with respect to the scalar and vector fields lead to the usual Euler-Lagrange equations. When applied to the Lagrangian (5), this gives

$$
\begin{gathered}
\square \omega_{a}-\nabla^{b} \nabla_{a} \omega_{b}-M^{2} \omega_{a}-g B_{a}=0, \\
\square \phi^{A}+\lambda \phi^{A}+\frac{g}{8 \pi} \epsilon^{a b c} \epsilon^{A B C}\left[3 \omega_{a} \nabla_{b} \phi_{B}+2 \phi_{B} \nabla_{b} \omega_{a}\right] \nabla_{c} \phi_{C} \\
=\frac{\delta V}{\delta \phi^{A}} .
\end{gathered}
$$

Solving for the Lagrangian multiplier, we get

$$
\lambda=\nabla^{a} \phi_{A} \nabla_{a} \phi^{A}+3 g \omega_{a} B^{a}+\frac{\delta V}{\delta \phi^{A}} \phi^{A}
$$

which, plugged into (7), leads to 


$$
\phi_{t t}^{A}=H_{D}^{A}\left\{\Delta \phi^{D}+\frac{g}{8 \pi} \epsilon^{a b c} \epsilon^{D B C}\left(3 \omega_{a} \nabla_{b} \phi_{B}+\phi_{B} F_{b a}\right) \nabla_{c} \phi_{C}+\left(\frac{\delta V}{\delta \phi^{B}} \phi^{B} \phi^{D}-\frac{\delta V}{\delta \phi_{D}}\right)\right\}-\phi^{A} \phi_{t}^{D} \phi_{D t}
$$

where $H_{B}^{A}=\delta_{B}^{A}-\frac{\phi^{A} \phi_{B}}{\phi^{2}}$ is the projector on the plane perpendicular to $\phi^{A}$. ${ }^{\phi}$

Note that the term $\frac{3 g}{8 \pi} \epsilon^{a b c} \epsilon^{A B C} \omega_{a} \nabla_{b} \phi_{B} \nabla_{c} \phi_{C}$ is parallel to $\phi^{A}$, so its projection will be zero and can be removed from the last equation.

From the remaining equation of motion (6), one can see that the mass $M$ breaks the gauge symmetry, enforcing the Lorentz gauge (i.e., $\nabla^{a} \omega_{a}=0$ ). Thus, the principal part of the $\omega_{a}$ equation is just the wave equation.

\section{Evolution equations}

In order to evolve the system, we bring it into a system of first order evolution equations. We choose a set of dynamical variables that we believe are the most convenient for doing this: For the scalar fields, we choose the fields themselves and their first derivatives, that is, $\phi^{A}$ and $\phi_{a}^{A} \equiv \partial_{a} \phi^{A}$. For the vector field, we consider $\omega_{a}, F:=$ $\frac{1}{2} \epsilon^{i j} F_{i j}$ and $E_{i}:=F_{i 0}$ (where $F_{a b}:=\nabla_{[a} \omega_{b]}$ is a lowdimensional analog of the electromagnetic tensor). We close the system by using the integrability condition for $F_{a b}$, namely, $\nabla_{[a} F_{b c]}=0$.

Thus, we obtain the following set of evolution equations:

$$
\begin{aligned}
\partial_{t} \phi_{t}^{A}= & H_{D}^{A}\left\{\frac{1}{\sqrt{-g}} \partial_{k}\left(\sqrt{-g} \phi_{k}^{D}\right)+\frac{g}{8 \pi} \epsilon^{a b c} \epsilon_{B C}^{D} \phi^{B} F_{b a} \phi_{c}^{C}\right. \\
& \left.+\left(\frac{\delta V}{\delta \phi^{B}} \phi^{B} \phi^{D}-\frac{\delta V}{\delta \phi_{D}}\right)\right\}-\phi^{A} \phi_{t}^{B} \phi_{t B} \\
\partial_{t} \phi_{i}^{A}= & \partial_{i} \phi_{t}^{A} \\
\partial_{t} E_{i}= & g^{j k} \nabla_{k}\left(\epsilon_{i j} F\right)+M^{2} \omega_{i}+g B_{i} \\
\partial_{t} \omega_{0}= & g^{i j} \nabla_{i} \omega_{j} \\
\partial_{t} F= & \epsilon^{i j} \partial_{j} E_{i} \\
\partial_{t} \omega_{i}= & \partial_{i} \omega_{0}-E_{i},
\end{aligned}
$$

subject to the two constraints

$$
\begin{gathered}
g^{j k} \nabla_{k} E_{j}-M^{2} \omega_{0}-g B_{0}=0, \\
F-\epsilon^{i j} \partial_{i} \omega_{j}=0 .
\end{gathered}
$$

It follows that the set of equations (9) constitutes a symmetric hyperbolic system [13], and thus, the Cauchy problem is well posed.
The energy density is found to be

$$
\begin{aligned}
T_{00}= & \frac{1}{2}\left[\dot{\phi^{A}} \dot{\phi_{A}}+\nabla_{k} \phi^{A} \nabla^{k} \phi_{A}+2 V(\vec{\phi})+E_{k} E^{k}+F^{2}\right. \\
& \left.+M^{2}\left(\omega_{0}^{2}+\omega_{k} \omega^{k}\right)\right] .
\end{aligned}
$$

It is important to note here that, although the interaction term does not appear explicitly in (12), it comes through the constraint (10) when minimizing the energy functional.

\section{Static solutions}

As mentioned, one purpose of this work is to find the static solutions of the model already introduced: the $\sigma$ model stabilized through the inclusion of a vector field.

A static configuration implies that all the spatial components of the baryonic current are zero because they involve temporal derivatives of the $\phi$ fields. Thus, given that the current $B^{i}$ acts as a source for $\omega_{i}$, one sees that the vector field $\omega_{i}$ should be constant and in fact must be null since it lives on the sphere. So $\omega_{i}=0$, and from now on, we will write $\omega_{0} \equiv \omega$ for notational convenience. In a static situation one also has, from the definitions, that $F=0$ and $E_{k}=\partial_{k} \omega$.

Performing the above substitutions on Eqs. (9)-(10), we get the following system of elliptic equations:

$$
\begin{gathered}
H_{D}^{A}\left\{\Delta \phi^{D}+\frac{g}{4 \pi} \epsilon^{D B C} \epsilon^{i j} \phi_{B}\left(\partial_{i} \omega\right)\left(\partial_{j} \phi_{C}\right)\right. \\
\left.+\left(\frac{\delta V}{\delta \phi^{B}} \phi^{B} \phi^{D}-\frac{\delta V}{\delta \phi_{D}}\right)\right\}=0, \\
\Delta \omega-M^{2} \omega-g B_{0}=0 .
\end{gathered}
$$

To solve these and get the static solutions, we evolve appropriate initial data along a parabolic flow given by

$$
\begin{gathered}
\partial_{t} \phi^{A}=H_{D}^{A}\left\{\Delta \phi^{D}+\frac{g}{4 \pi} \epsilon^{D B C} \epsilon^{i j} \phi_{B}\left(\partial_{i} \omega\right)\left(\partial_{j} \phi_{C}\right)\right. \\
\left.+\left(\frac{\delta V}{\delta \phi^{B}} \phi^{B} \phi^{D}-\frac{\delta V}{\delta \phi_{D}}\right)\right\}, \\
\partial_{t} \omega=\Delta \omega-M^{2} \omega-g B_{0} .
\end{gathered}
$$

These are essentially heat-type equations (parabolic system) but with some extra terms. We note that if the evolution of such a system dissipates energy and reaches a stationary state, then the right-hand sides of (15) and (16) would be zero and we would be in the presence of a solution of (13) and (14), namely, a static solution of the theory. 


\section{COMPUTATION}

In this study we have used two different codes. One implements the full dynamics of the system through the computation of the hyperbolic set of equations (9), while the other serves to explore the possible static configurations by evolving the parabolic system (15) and (16). The key feature of having these two codes relies on the possibility of using the output of one of them as initial data for the other. This allows us to check, in a fully dynamical setting (hyperbolic code), if a given configuration found after the diffusion process (parabolic code) is really a static and stable solution of the theory.

\section{A. Grid scheme}

As mentioned, the topology of our computational domain is $S^{2}$, the unit sphere. Since it is not possible to cover the whole sphere with a single system of coordinates which is regular everywhere, we employ multiple patches to cover it.

A convenient set of patches is defined by the cubed sphere coordinates. There are six patches with coordinates projected from the sphere, and each of these patches constitutes a uniform grid, with a variable amount of points (see Fig. 1).

These grids are defined in such a way that there is no overlap, and only grid points at the boundaries are common to different grids (multiblock approach).

It is evident that to solve a problem under this grid structure, one must ensure the suitable transfer of information among the different grids. We basically follow the technique described in [14], which relies on the addition of suitable penalty terms to the evolution equations $[15,16]$. These terms penalize the possible mismatches between the different values that the characteristic fields take at the interfaces. For the parabolic case, they were obtain from an extension to the two-dimensional case by Parisi-Reula (work in progress) from the studies of Carpenter et al. $[15,16]$.

\section{B. Numerical scheme and stability}

In order to construct stable finite difference schemes for our initial value problems, we use the method of lines [13].
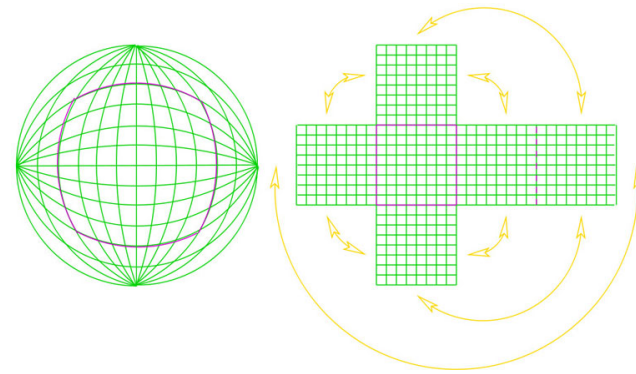

FIG. 1 (color online). Cubed sphere coordinates. A total of six Cartesian patches are employed to cover the sphere. Only patch boundaries coincide at common points.
This means that we first discretize the spatial derivatives (constructing some finite difference operators) so as to obtain a large system of ordinary differential equations for the grid functions. This system is usually called a semidiscrete system.

To ensure the stability of the numerical scheme, we use the energy method described in [17]. First, one has to check that the initial value problems are well posed at the continuous level and that the solutions of the partial differential equations satisfy an energy estimate which bounds some norm of the solution. Then, one constructs a difference operator that satisfies summation by parts (the discrete analogue of integration by parts) in the discrete version of that norm. This operator, together with the appropriate penalty terms at the interfaces, implies a semidiscrete energy estimate which ensures that the system is stable. Finally, by discretizing the time derivatives, one obtains the fully discrete system which is numerically implemented. If the semidiscrete system is stable, one can show that the fully discrete system is stable as well, provided that an appropriate time integrator is chosen. We have used, for both codes, a classical fourth order Runge-Kutta method. For the parabolic code one can use much simpler, although less accurate, time discretizations, in particular, Euler's method.

We have backed up all our numerical results by monitoring conserved quantities like the energy and the topological charge.

\section{Initial data}

The initial data were constructed from rational maps, which are the well-known static solutions of the sigma model (solutions of the Bogomolny equations). (For a review on the subject see [18].)

Rational maps of each topological sector were used to set the initial values for the scalar field. Written in terms of the target space coordinate $R=R(z, \bar{z})$, where $R=\left(\phi_{1}+\right.$ $\left.i \phi_{2}\right) /\left(1+\phi_{3}\right)$ (and with $z=\tan \frac{\theta}{2} e^{i \varphi}$ given in standard angular coordinates), the map used was $R(z)=\frac{\lambda}{z^{B}}$, with $\lambda \in \mathbb{R}$ and $B$ being the topological degree of the map. These configurations represent rings of baryon density for all values of charge $B \geq 2$.

For the vector meson field, we approximate its initial values with $\omega=\frac{g}{M^{2}} B^{0}$, which comes from neglecting the Laplacian in Eq. (14).

As mentioned, for the code that runs the full dynamics (hyperbolic), we use the output configurations attained with the previous diffusion process (parabolic), setting the initial values of the remaining dynamical variables (i.e., the absent fields in the parabolic equation) to their static values. That is, $\partial_{t} \phi^{A}=0, F=0$ and $E_{k}=\partial_{k} \omega$.

\section{RESULTS}

\section{A. Without potential $(V=0)$}

While in flat two-dimensional space a potential term $V(\vec{\phi})$ is mandatory to ensure the existence of stable 
solutions, in the present model it is not. The need for the inclusion of this potential comes from the necessity of avoiding Derrick's scaling argument [19], and it basically determines, together with the other terms added to the Lagrangian of the sigma model, the appearance of a preferred size for the solitons. In the case of the unit sphere, however, there already exists a natural spatial scale: the radius of curvature. Therefore, Derrick's argument does not apply in this case, and stable static solutions are, in principle, allowed.

Thus, it is expected that the solitons, in the absence of a potential term, will be spread out over the entire space. And, in particular, the $B=1$ solution is expected to be the uniform energy (or topological) density distribution on the sphere. This is in fact the case, and it corresponds simply to the rational map solution $R(z)=\frac{1}{z}$, which is found to satisfy the static equations (13) and (14), leading to the configuration $B^{0}=\frac{1}{4 \pi}=$ cte. We were able to confirm numerically that this is the static configuration of the sector $B=1$, and to verify that the energy found agrees with its theoretical value given by $E=4 \pi+\frac{1}{4 \pi} \frac{g^{2}}{M^{2}}$.

The sector $B=1$ seems to be the only topological sector in which the full $\mathrm{O}(3)$ symmetry of the theory is preserved. The $B=2$ solution turned out to be axially symmetric [corresponding to the $\mathrm{O}(2)$ subgroup], whereas higher charge multisolitons were all found to have point symmetries which are subgroups of $\mathrm{O}(3)$. In order to ensure these results are not influenced by the initial data chosen (on the first two sectors) or by the grid structure employed (on the higher charged cases), we have included an artificial numerical perturbation on top of the relaxation scheme (parabolic code). This mechanism consists in the addition, to every field at every grid point and for each time step, of a random number chosen in the range $(-1,1)$ multiplied by a coefficient $\delta$ (generally chosen to be $\delta=10^{-3}$ ). Such a mechanism has shown the robustness of the axial symmetry in the $B=2$ sector and gives us more confidence that the configurations found are not influenced by the numerical implementation. In addition, we have observed that it seems to enhance the diffusion process as well.

In Ref. [12] an intimate relation was pointed out between the baby-Skyrme model on the sphere and the 3D Skyrme model. The symmetries of the 3D Skyrme model are determined solely by the angular dependence of the Skyrme field, and it was suggested that this 2D version may be thought of as the 3D Skyrme model with a "frozen" radial coordinate. They were able to demonstrate the connection within the rational map approximation and show full field simulations for charges up to $B=14$ having the same symmetries as corresponding solutions of the $3 \mathrm{D}$ Skyrme model.

We present in Fig. 2 the static configurations found within our numerical scheme, for charges $2 \leq B \leq 16$, for the vector meson stabilization of the sigma model. Our plots are incredibly similar to those in [12], they and show

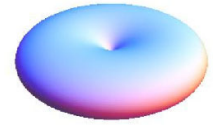

(a) $B=2$

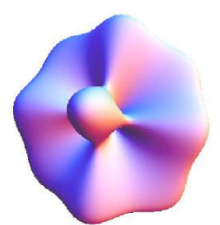

(d) $B=5$

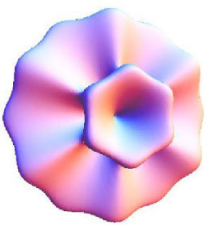

(g) $B=8$

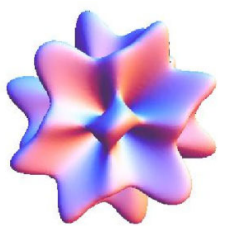

(j) $B=11$

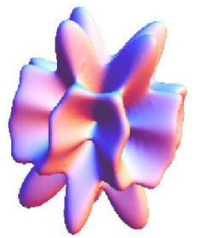

(m) $B=14$

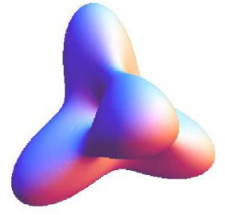

(b) $B=3$

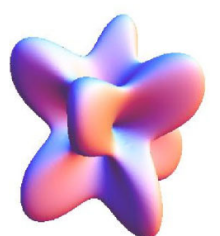

(e) $B=6$

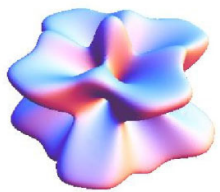

(h) $B=9$

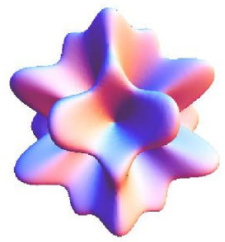

(k) $B=12$

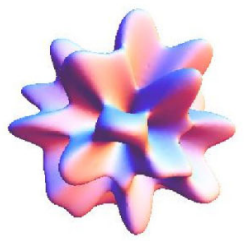

(n) $B=15$

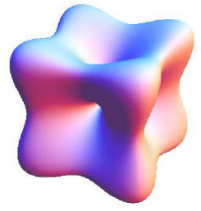

(c) $B=4$

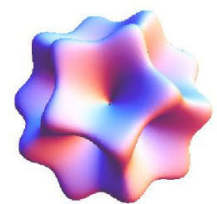

(f) $B=7$

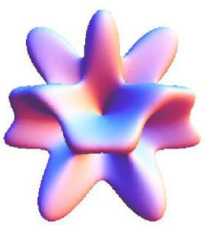

(i) $B=10$

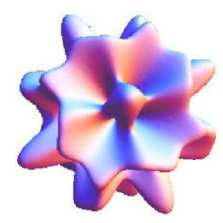

(1) $B=13$

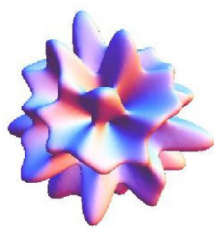

(o) $B=16$
FIG. 2 (color online). $V=0$. Multisolitons for charges $2 \leq B \leq 16 .\left(g=18 ; M \approx 6.4 \rightarrow \kappa^{2}=0.05\right)$.

once again the great resemblance of the two models. The calculations were done for several values of the parameters $g$ and $M$, yielding qualitatively similar configurations within each topological sector. Indeed, quantitative similarities also follow (e.g. see Table I).

The expressions for the static energy of the two models are

$$
\begin{array}{r}
E_{\text {static }}^{\mathrm{B}-\mathrm{S}}=\frac{1}{2} \int_{S^{2}} \epsilon\left[(\nabla \phi)^{2}+\kappa^{2}(4 \pi)^{2}\left(B^{0}\right)^{2}\right], \\
E_{\text {static }}^{\mathrm{V}-\mathrm{M}}=\frac{1}{2} \int_{S^{2}} \epsilon\left[(\nabla \phi)^{2}+\frac{g}{M^{2}} B^{0} \Delta \omega+\frac{g^{2}}{M^{2}}\left(B^{0}\right)^{2}\right] .
\end{array}
$$

In [5] an approximation of the solutions of (14) was suggested by applying a derivative expansion, in which at first order the Laplacian is neglected. So the energies in 
TABLE I. Energies and symmetries of the vector meson and baby-Skyrme models for $\kappa^{2}=\left(\frac{g}{4 \pi M}\right)^{2}=0.05$ (with $g=18$, $M \approx 6.4)$.

\begin{tabular}{lcccc}
\hline \hline \multirow{2}{*}{$\begin{array}{c}\text { Charge } \\
\text { B }\end{array}$} & \multicolumn{2}{c}{ Baby Skyrme } & \multicolumn{2}{c}{ Vector meson } \\
& $E / 4 \pi B$ & Symmetry & $E / 4 \pi B$ & Symmetry \\
\hline 2 & 1.071 & $D_{\infty h}$ & 1.068 & $D_{\infty h}$ \\
3 & 1.105 & $T_{d}$ & 1.099 & $T_{d}$ \\
4 & 1.125 & $O_{h}$ & 1.117 & $O_{h}$ \\
5 & 1.168 & $D_{2 d}$ & 1.155 & $D_{4 d}$ \\
6 & 1.194 & $D_{4 d}$ & 1.179 & $D_{4 d}$ \\
7 & 1.209 & $I_{h}$ & 1.193 & $I_{h}$ \\
8 & 1.250 & $D_{6 d}$ & 1.229 & $D_{6 d}$ \\
9 & 1.281 & $D_{4 d}$ & 1.256 & $D_{4 d}$ \\
10 & 1.306 & $D_{4 d}$ & 1.278 & $D_{4 d}$ \\
11 & 1.337 & $D_{3 h}$ & 1.306 & $D_{3 h}$ \\
12 & 1.360 & $T_{d}$ & 1.328 & $T_{d}$ \\
13 & 1.386 & $O_{h}$ & 1.352 & $O_{h}$ \\
14 & 1.421 & $D_{2}$ & 1.383 & $D_{4 d}$ \\
\hline \hline
\end{tabular}

Data taken from [12].

the two models could be compared upon identification of the parameters as $\kappa=\frac{g}{4 \pi M}$. Therefore, we chose our parameters $g$ and $M$ appropriately for comparison of the energies found in the present study for the vector meson theory with the ones obtained in [12] for the baby-Skyrme model with $\kappa^{2}=0.05$. The energies (normalized with $4 \pi B$ ) for both models are listed in Table I, for charges up to $B=14$.

It can be seen from Table I that the energies are very similar in both models, with the energy of the vector meson theory only slightly smaller than the ones found in [12] for the baby-Skyrme case.

The symmetries we find in our solutions generally agree with those in [12], with only a minor discrepancy in the $B=5$ sector and a more significant one in the $B=14$ solution, where we have found a more symmetrical configuration. It is difficult to argue whether these $B=14$ solutions are different simply because different models are being compared or because one of them is only a local minimum of the energy.

We note here that the symmetries of the multisoliton solutions we have found match exactly (up to charge 13) with the symmetry group $H_{B}$ of $2 B-2$ point Coulomb charges on a sphere minimizing the energy, known as the Thomson problem. (See Ref. [20] for more details.)

A final comment on Table I is necessary. As we have mentioned, we fixed the ratio $\frac{g}{M}$ for comparison between the two models. Hence, there is still freedom remaining in setting the values $g$ and $M$ independently, and we have observed that the energies of the two models approach each other for larger values of both parameters, being significantly smaller in the vector meson theory when small values for $g$ or $M$ are considered.

The fact that the energies for both theories approach each other for large $g$ and $M$ is reasonable in view of Eq. (18), and the smaller values attained when $g$ and $M$ are small suggest the possibility of the second term of this energy expression taking negative values.

\section{B. With potential $(V \neq 0)$}

The spatial scale adopted by the solitons in the previous section with $V=0$ is the natural length scale provided by the radius of curvature of the sphere. The inclusion of a potential term is known to endow the solitons with a preferred size that depends on the parameters of the theory (and not on the geometry). So, there will be two length scales, namely, the size of the solitons and the radius of curvature of the unit sphere.

However, the choice for the potential is largely arbitrary (given that any potential which contains no field derivatives would do equally well), and it has been seen that the form of this term has a major impact on the existence and structure of baby multi-Skyrmions [21,22].

In this first exploratory work, we shall use the potential term

$$
V(\vec{\phi})=m^{2}\left(1-\phi_{3}\right)
$$

This particular potential form is motivated by analogy with the one traditionally used in the three-dimensional Skyrme model, and the suggestive name given to the parameter comes from the idea of a mass term for the pion field. Note that the inclusion of this term breaks the $\mathrm{O}(3)$ symmetry of the model, leaving only the $\mathrm{O}(2)$ subgroup.

We have three parameters in our model, namely, the "pion mass" $m$, the "vector meson mass" $M$, and the coupling constant $g$. We shall identify two useful combinations $\alpha$ and $\beta$, which help us to organize the exploration through the parameter space. The first one, $\alpha:=\left(\frac{m g}{M}\right)$, is a dimensionless quantity representing the interaction strength with the vector meson field, while $\beta:=\left(\frac{m M}{g}\right)^{-1 / 2}$ gives the ratio between the length scale of the solitons and the length scale of the target space (its curvature radius), and one might regard it as a sort of soliton density. Notice that these are not new parameters but rather useful combinations (especially $\beta$ ) which will allow us to classify static configurations.

Below we present the static solutions found for the topological sectors $1 \leq B \leq 9$, obtained after the diffusion process discussed previously, namely, the evolution of the parabolic system (15) and (16).

To display them in this work we have chosen to plot the topological density $B^{0}$. Notice that, for all the cases we have considered, the plots associated with the energy density are very similar to these ones.

In the topological sectors with charge $B=1$ and $B=2$, we have found static solutions that are axially symmetric in all the cases. They preserve the whole $\mathrm{O}(2)$ subgroup. In the sector of topological charge 1, these solutions represent lumps of energy (and topological charge), whose sizes are 


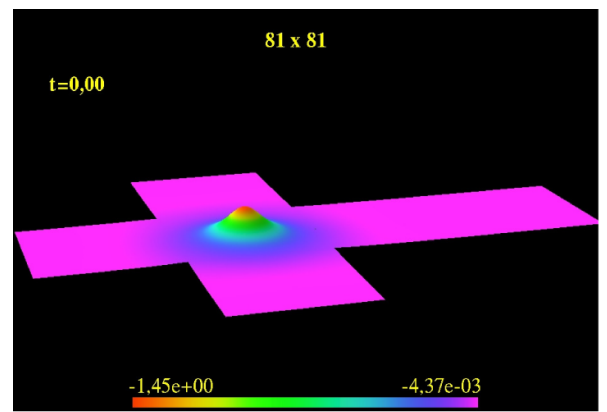

(a)

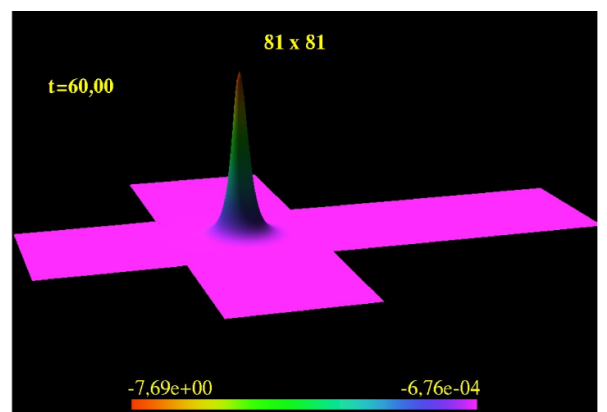

(b)

FIG. 3 (color online). $\quad B=1$. Initial and final configurations $\left(g=2, M^{2}=16, m^{2}=0,1\right)$.

in correspondence with the parameters employed. (See Fig. 3.) When $\beta$ increases, the soliton spreads out in space until it reaches a uniform distribution in the $\beta \rightarrow \infty$ limit, the one previously presented in the $V=0$ case.

In the sector $B=2$, instead, we have found ringlike configurations. The radii of these rings are of course related to the parameters, and again $\beta$ seems to be the most relevant among them. For small values of $\beta$ one sees the rings localized around one of the poles, while for large values, the radius increases and approaches the equator. (See Fig. 4.)

All these solutions happen to be stable when introduced as initial data for the hyperbolic code, in which the full dynamics of the model is tested, even if a perturbation is introduced into the initial setup. In the case of the rings, for example, if one introduces a small radial perturbation, the ring oscillates back and forth around an equilibrium position.

In topological sectors of higher charges $(B>2)$, we have found a rich variety of static solutions according to the parameters used. There are no axially symmetric configurations anymore. Despite starting with axially symmetric initial data, during the relaxation procedure (parabolic code) this symmetry eventually breaks down. We have noticed that, originally, this symmetry breaking took place at the interfaces of our grid structure, where less accuracy from the finite difference operators was expected. Thus, in order to avoid, as much as possible, the influence of the grid infrastructure on the system's behavior, we have also included the numerical perturbation discussed in Sec. IV A.

The multisoliton solutions can be arranged basically in two regimes: a low density $(\beta \lesssim 2)$ and a high density $(\beta \gtrsim 3)$ phase. The transition between these two regimes does not seem to be sharply defined. As noted in [23], it is not a phase transition in the usual sense, and this is partly due to the asymmetry of the system (because of the potential term used).

The main qualitative difference between the two regimes is that, in the first one $(\operatorname{small} \beta)$, the solitons are localized in space, grouped generally in pairs and individually, while in the high density phase (large $\beta$ ), the solitons are spread over the whole sphere forming very structured configurations.

\section{Small $\beta$ regime}

Clearly, the (two-dimensional) plane case is meant to be the limit in which $\beta=0$ and $\alpha \propto g$. Thus, in this regime of small values for $\beta$, the configurations are expected to be localized and to share many of the features present in the flat-space studies, such as [21,22,24,25] for baby Skyrmions or [5] for the (2D) vector meson theory.

Although, of course, we cannot reach the limit $\beta=0$ (which would imply infinite resolution on the grids), we were able to find configurations localized enough to reflect these properties.

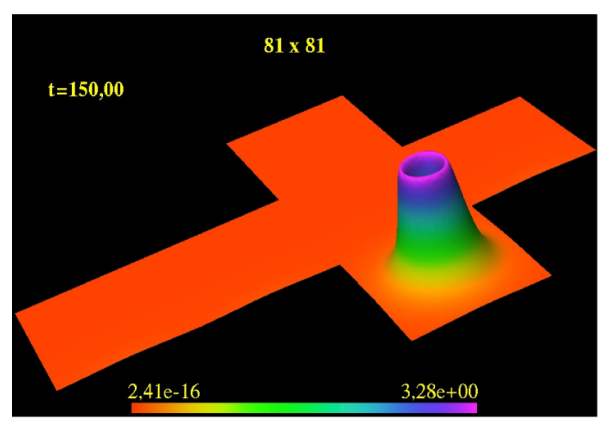

(a) $\beta=1.06$

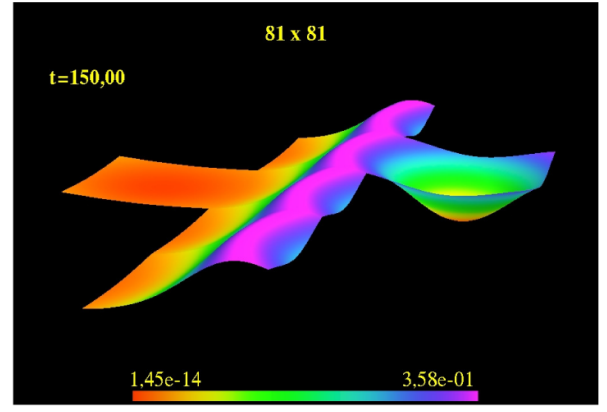

(b) $\beta=3$

FIG. 4 (color online). $\quad B=2$. Static configurations for different densities $\beta$. 


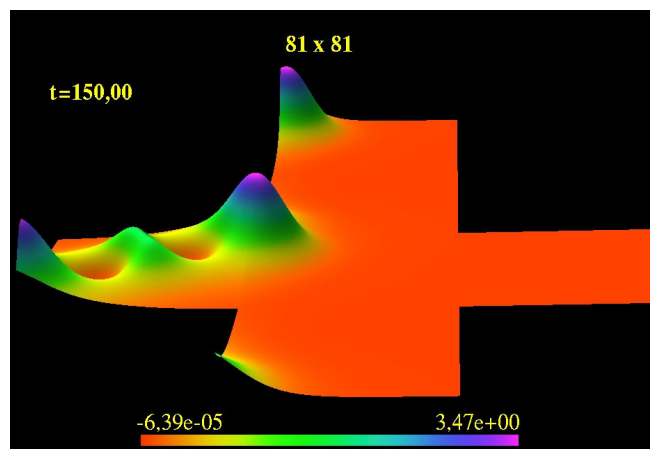

FIG. 5 (color online). $B=3$. Low density three-soliton ( $\alpha=1.58 ; \beta=1.26)$.

For $B=3$, our numerical procedure leads to configurations like the one displayed in Fig. 5, where the solution is made up of three distorted solitons aligned and binded together. This is the well-known three-soliton that appears in all the studies mentioned above, including [5] in which the similarities of the two models were established.

For higher sectors $(B \geq 4)$, the baryon density plots in Fig. 6 show the configurations obtained for one of the several parameters explored for values close to $\beta=1$, all presenting the same qualitative features.

The plots suggest that the two-soliton serves as a basic building block for higher multisolitons, especially for those sectors with an even topological charge. As pointed out in [21], the two-soliton interaction seems to be the energetically most favorable breakup mode. We have observed this behavior during the evolution of Eqs. (15) and (16) (diffusion process). In the cases where $B$ is odd, the system breaks up into soliton pairs and one individual soliton. After the breakup, the constituents start to attract each other to form structured states. However, these states appear to be very weakly bound and exist, in the literature, in different possible combinations of the individuals, two-soliton, and

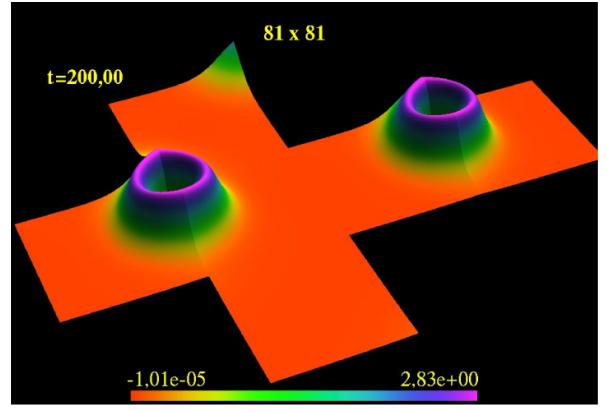

(a) $B=4$

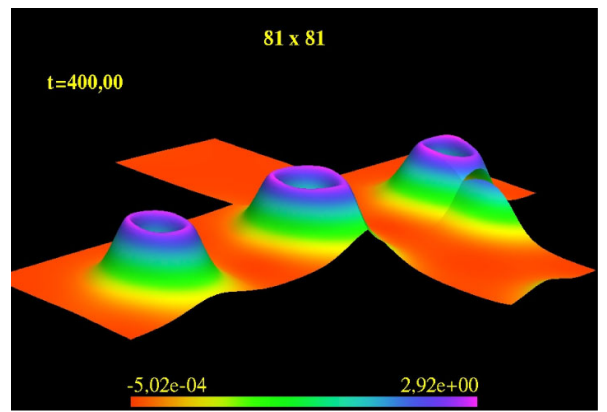

(c) $B=6$

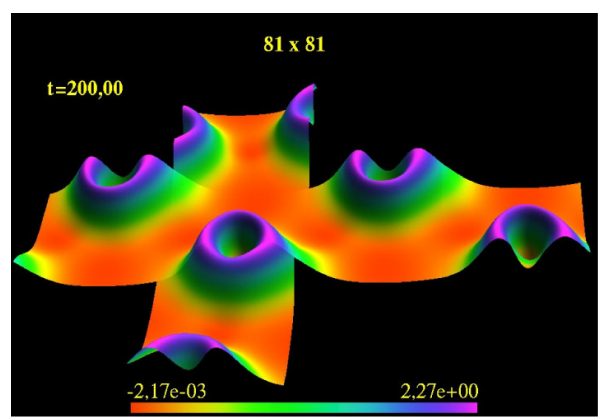

(e) $B=8$

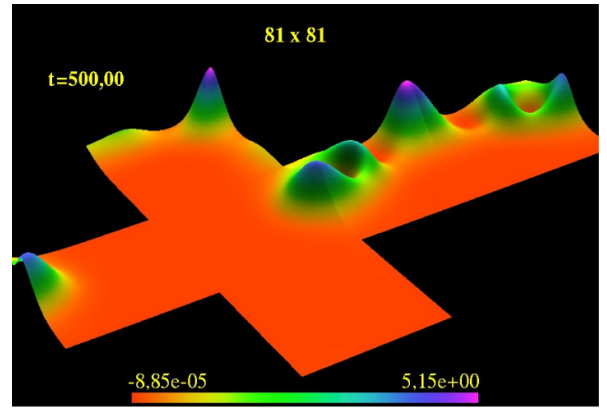

(b) $B=5$

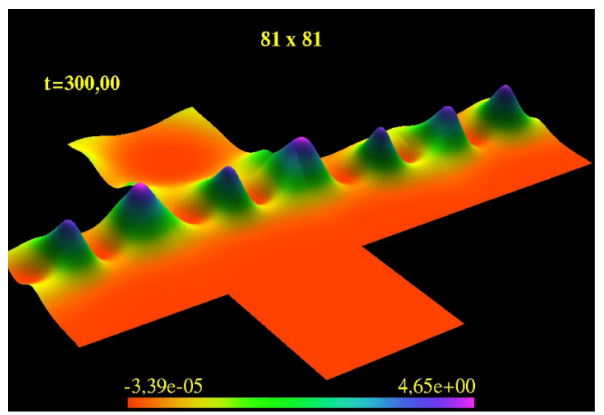

(d) $B=7$

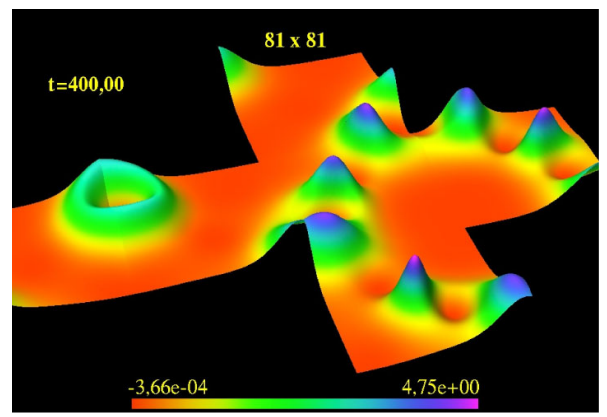

(f) $B=9$

FIG. 6 (color online). Low density multisolitons of degree $4 \leq B \leq 9(\alpha=4.24 ; \beta=1.03)$. 
TABLE II. Energies of localized multisolitons in the V-M theory $(\alpha=4.24 ; \beta=1.03)$.

\begin{tabular}{lccl}
\hline \hline $\begin{array}{l}\text { Charge } \\
\mathrm{B}\end{array}$ & $\begin{array}{c}\text { Energy } \\
E\end{array}$ & $\begin{array}{c}\text { Energy per soliton } \\
E / 4 \pi B\end{array}$ & Description \\
\hline 1 & 18.4 & 1.47 & Individual \\
2 & 35.9 & 1.43 & Ring \\
3 & 54.2 & 1.44 & Three-soliton \\
4 & 71.9 & 1.43 & Two rings \\
5 & 90.0 & 1.43 & Open chain \\
6 & 107.8 & 1.43 & Three rings \\
7 & 125.8 & 1.43 & Closed chain \\
8 & 143.8 & 1.43 & Four rings \\
9 & 161.8 & 1.43 & Ring + closed chain \\
\hline \hline
\end{tabular}

three-soliton constituents, forming various crystal-like patterns within each topological sector. Also, there is one further type of multisolitonic solutions, first proposed in [25], consisting of Skyrmion chains.

Clearly, the decision on whether a given configuration is a local or a global minimum of the energy becomes subtle in this context. For that reason, and since we are just recreating the planar case in the sphere (adjusting the parameters of the model so as to localize the solutions), we are not in the position to contribute much to the above discussion. Instead, we present the static configurations obtained up to charge $B=9$ (Fig. 6) and list the corresponding energies in Table II, reinforcing once again the similitudes between the vector meson stabilization of the sigma model and the more traditional baby-Skyrme model.

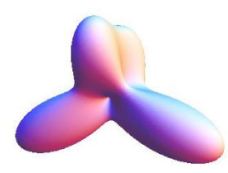

(a) $B=3\left(D_{2 d}\right)$

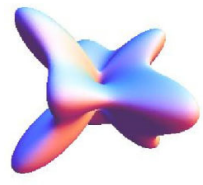

(c) $B=5\left(D_{2 d}\right)$

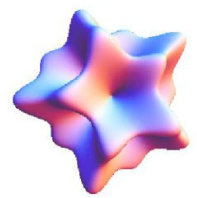

(e)

$B=7\left(D_{5 d}\right)$

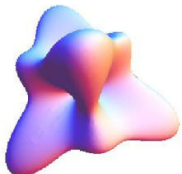

(b)

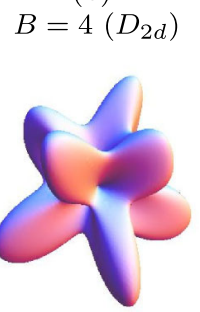

(d) $B=6\left(D_{2 d}\right)$

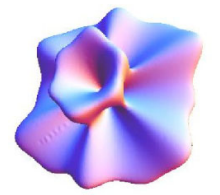

(f)

\section{Large $\beta$ regime}

For higher densities, starting from $\beta$ values close to 3 , we find structured solutions like the ones shown in Fig. 2. This should not come as a surprise, since the $V=0$ case represents a limit in which $\beta$ goes to infinity.

For the smallest values of $\beta$ within this regime, we find multisolitonic solutions like the ones we display in Fig. 7 for the sectors $3 \leq B \leq 11$. Notice that they are distorted versions of the topological density plots of Fig. 2.

It is important to recall here that the potential term included breaks the $\mathrm{O}(3)$ symmetry of the solutions into the $\mathrm{O}(2)$ subgroup. So in this case, the configurations have symmetries which are discrete subgroups of $\mathrm{O}(2)$, rather than $\mathrm{O}(3)$. This is clearly seen in the solutions of the sectors $B=3, B=4$, and $B=7$, having platonic symmetries when $V=0$ (tetrahedral, cubic and icosahedral, respectively), and now exhibiting dihedral symmetries with a preferred axis.

For larger values of $\beta$, these effects become weaker and the multisolitonic solutions approach those of vanishing potential already discussed in the previous section.
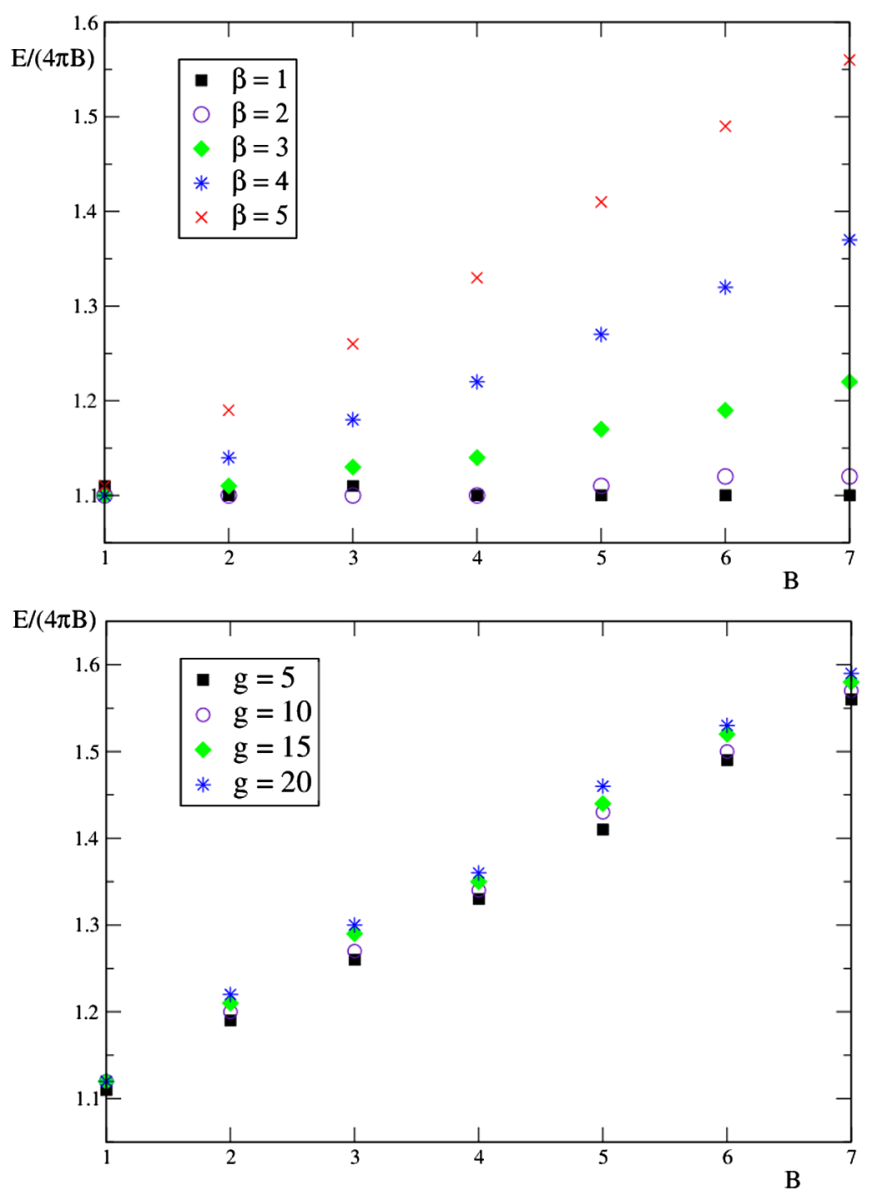

FIG. 8 (color online). Energy (per soliton) dependence with topological charge. Top panel: Different values $\beta$ for fixed $\alpha=1, g=5$. Bottom panel: Different values $g$ for fixed $\alpha=1$, $\beta=5$.
FIG. 7 (color online). High density multisolitons for charges $3 \leq B \leq 8$, with their symmetry groups $(\alpha=1.8, \beta=3$; $\kappa^{2} \simeq 0.1, m^{2}=0.2$ ). 


\section{Energy dependence}

Here, we present a study of the energy dependence of the static solutions with the parameters $(\alpha, \beta, g)$. In Fig. 8 we display the energy per soliton as a function of the topological charge for different values of $\beta$ (top image) and $g$ (bottom image), while keeping all the other parameters constant. From these figures, it can be seen that the energy per soliton grows approximately linearly with the topological charge, since the slope of these curves is related to the solitonic density $\beta$ : For small values of $\beta$ the energy per soliton remains almost constant, while for higher values, it begins to grow with the soliton charge. The coupling parameter $g$, on the other hand, seems to leave the slopes fixed but slightly displaces the curves upwards when increasing its value.

\section{SUMMARY AND CONCLUSIONS}

We have studied the vector meson stabilization of the sigma model on the two-sphere, performing a numerical implementation of the problem which allows us to find static multisoliton solutions and to dynamically check whether such solutions are stable or not.

We were able to compare our solutions up to charge $B=14$, for the massless pion case $(V=0)$, with the ones obtained in the baby-Skyrme model, finding an incredible correspondence between the two models, not only on a qualitative level (symmetries) but also on a quantitative level (energies). These solutions generally were found to have the same symmetries as the corresponding multiSkyrmions of the 3D Skyrme model, and a strong connection between them was suggested in the literature. We did not pursue these arguments further to explore the possible effects that a nonzero pion mass would have because we believe this association between the twodimensional version on the sphere and the threedimensional model is only true for solutions that can be well approximated by rational maps (which was the case here); however, the introduction of the potential term will modify this situation, and a more careful analysis would be required. We defer such an analysis to a forthcoming work, in which we will be interested in the possibility of extending the studies $[26,27]$ to the theory with vector mesons and no Skyrme term. In those studies, it is shown that there is an important qualitative difference between multi-Skyrmions (in the standard Skyrme model) with massive or massless pions. For sufficiently large pion mass or baryon number, the structures collapse to form qualitatively different stable Skyrmion solutions.

We have also explored here the inclusion of a potential term (the traditional option, corresponding to a pion mass analogue) and the interplay generated between the new length scale it introduces and the natural spatial scale of the two-sphere. We have identified the relevant parameter associated with the concept of a soliton density in space, and we have basically observed two regimes in which very different types of solutions are found. However, the transition is not sharply defined. The first regime is a low density phase where we have found localized solutions that allowed us to recreate the planar two-dimensional case on the sphere, hence allowing us to compare our solutions with the ones presented in the literature (both for baby-Skyrmion and vector meson stabilization), finding good qualitative agreement.

The second regime of higher soliton densities shows structured solutions covering the entire sphere that are very similar to those encountered for the massless $(V=0)$ case. They seem to be distorted and to lose their symmetry when close to the phase transition $(\beta \sim 3)$.

It is worth mentioning that we already have the numerical infrastructure to study the dynamical aspects of the model as well, in particular, the scattering and annihilation process like the ones presented in Refs. [6,7], where a rich variety of phenomena and an intimate connection with the Skyrme model have been found.

\section{ACKNOWLEDGMENTS}

The authors wish to acknowledge the assistance of the Consejo Nacional de Investigaciones Científicas y Técnicas (CONICET) and the Universidad Nacional de Córdoba, both of which support facilities used in this investigation. This work is supported by Projects No. FONCYT PICT-Bicentenario-2010-1387, No. PICT-Raices-20081593, No. CONICET PIP-11220080100754, and SeCyT, Universidad Nacional de Córdoba, No. 05/B415.
[1] T. H. R. Skyrme, Proc. Roy. Soc. Lond., Ser. A 260, 127 (1961).

[2] E. Witten, Nucl. Phys. B160, 57 (1979).

[3] G. S. Adkins and C. R. Nappi, Phys. Lett. B 137, 251 (1984).

[4] P. Sutcliffe, Phys. Rev. D 79, 085014 (2009).

[5] D. Foster and P. Sutcliffe, Phys. Rev. D 79, 125026 (2009).
[6] R. D. Amado, M. A. Halasz, and P. Protopapas, Phys. Rev. D 61, 074022 (2000).

[7] M. A. Halasz and R. D. Amado, Phys. Rev. D 63054020 (2001).

[8] N. S. Manton, Commun. Math. Phys. 111, 469 (1987).

[9] N. S. Manton and P. J. Ruback, Phys. Lett. B 181, 137 (1986). 
[10] S. L. Sondhi, A. Karlhede, S. A. Kivelson, and E. H. Rezayi, Phys. Rev. B 47, 16419 (1993).

[11] A. A. Belavin and A. M. Polyakov, JETP Lett. 22, 445 (1975).

[12] I. Hen and M. Karliner, Phys. Rev. E 77, 036612 (2008).

[13] B. Gustafsson, J. Oliger, and H. Kreiss, Time Dependent Problems and Difference Methods (John Wiley and Sons, New York, 1995).

[14] L. Lehner, O. Reula, and M. Tiglio, Classical Quantum Gravity 22, 5283 (2005).

[15] M. H. Carpenter, J. Nordström, and D. Gottlieb, J. Comput. Phys. 148, 341 (1999).

[16] J. Nordström and M. H. Carpenter, J. Comput. Phys. 173, 149 (2001).

[17] G. Calabrese, L. Lehner, O. Reula, O. Sarbach, and M. Tiglio, Classical Quantum Gravity 21, 5735 (2004).
[18] N. Manton and P. Sutcliffe, Topological Solitons (Cambridge University Press, Cambridge, England, 2004).

[19] G. H. Derrick, J. Math. Phys. (N.Y.) 5, 1252 (1964).

[20] R. Battye, C. Houghton, and P. Sutcliffe, J. Math. Phys. (N.Y.) 44, 3543 (2003).

[21] T. Weidig, Nonlinearity 12, 1489 (1999).

[22] P. Eslami, M. Sarbishaei, and W. Zakrzewski, Nonlinearity 13, 1867 (2000).

[23] M. de Innocentis and R. S. Ward, Nonlinearity 14, 663 (2001).

[24] B. M. A. G. Piette, B. J. Schroers, and W. J. Zakrzewski, Z. Phys. C 65, 165 (1995).

[25] D. J. Foster, arXiv:0904.3846.

[26] R. Battye and P. Sutcliffe, Nucl. Phys. B705, 384 (2005).

[27] R. Battye and P. Sutcliffe, Phys. Rev. C 73, 055205 (2006). 